\section{The National Early Warning Score 2 (NEWS2) - Elderly patients and training of nursing / allied healthcare professionals in using NEWS2}

Editor - I read with interest, 'The inclusion of delirium in version 2 of the National Early Warning Score will substantially increase the alerts for escalating levels of care: findings from a retrospective database study of emergency medical admissions in two hospitals' by Mohammed et al and 'The National Early Warning Score and the acutely confused patient' ${ }^{2}$ by Bryan Williams. Being a geriatric medicine and general internal medicine higher specialist trainee, who has been working at the front lines and in the medical/surgical wards, my experiences resonate with the facts described in the above-mentioned articles. Although appearance of new confusion necessitates escalation of care among patients ( $\geq 85$ years of age) with infection, there was a high prevalence of patients in this age group who did not have any evidence of underlying infection coinciding with development of new confusion. ${ }^{3}$ Infection is a major cause of delirium ${ }^{4}$ but other causes are as important to be considered and dealt with as well. ${ }^{5}$ In addition to the challenges described by Mohammed et al, ${ }^{1}$ one important issue to be recognised is staff (nursing and allied healthcare professionals (AHPs)) training to allow them to recognise delirium / new onset confusion in the elderly population (which is currently the main population cohort requiring medical attention) with or without background cognitive impairment. There must be a validated tool which nurses and AHPs should be able to use to recognise delirium I new confusion and then score that on the NEWS2 scale. One systematic review looked at 21 delirium screening tools described in 31 studies and found that confusion assessment method was the most used tool, with delirium rating scale giving best results in screening for delirium / new confusion. ${ }^{6}$ Both screening tools require teaching and training of staff using them for optimal results. ${ }^{6}$ Therefore, in order to streamline NEWS2's implementation, decrease false positive alerts/escalations and improve its outcomes, we must train our nursing/AHP staff to use a validated screening tool to identify delirium / new confusion. And for this purpose, we must utilise the expertise of our geriatric medicine colleagues (physicians, clinical nurse specialists and registered nurses).

BILAL HAIDER MALIK ST4 geriactric medicine and general internal medicine, Colchester, UK

\section{References}

1 Mohammed MA, Faisal M, Richardson D et al. The inclusion of delirium in version 2 of the National Early Warning Score will substantially increase the alerts for escalating levels of care: findings from a retrospective database study of emergency medical admissions in two hospitals. Clin Med 2019:19:104-8.

2 Williams B. The National Early Warning Score and the acutely confused patient. Clin Med 2019;19:190-1.

3 Camm CF, Hayward G, Elias TCN et al. Sepsis recognition tools in acute ambulatory care: associations with process of care and clinical outcomes in a service evaluation of an Emergency Multidisciplinary Unit in Oxfordshire. BMJ Open 2018;8:e020497.

4 Ferrer R, Martin-Loeches I, Phillips G et al. Empiric antibiotic treatment reduces mortality in severe sepsis and septic shock from the first hour: results from a guideline-based performance improvement program. Crit Care Med 2014;42:1749-55.

5 National Institute of Health and Care Excellence. Sepsis: recognition assessment and early management. NICE guideline [NG51]. London: NICE, 2016
6 De J, Wand APF. Delirium screening: A systematic review of delirium screening tools in hospitalized patients. Gerontologist 2015;55:1079-99.

\section{Acute neurology service}

Editor - We read the article 'Hyperacute neurology at a regional neurosciences centre' with interest. 'The experience of the St George's team in their first year of providing the service demonstrates the improvements in care that can be anticipated through the intensive involvement of neurology at the 'front door'.

Our own experience also supports the approach (though we use the term 'acute neurology service'). We hope this may also be of interest to units as it demonstrates that such systems can be both 'scaled up' and become embedded as a 'routine' method of care. We have been running a 365 days-a-year, 7 days-a-week acute neurology service in Plymouth for almost 15 years. Last year we were proud to be finalists in the Health Service Journal awards for best Acute Services Redesign.

We found that this method of care reduced length of stay by $37 \%$ and the bed base by $46 \%$. When we analysed the first 10 years of our service we found we had cared for approximately 20,000 patients. Our acute neurology admission avoidance clinics have been running for around 6 years and have cared for approximately 4,500 patients. They have mitigated the effects of the sustained rise of acute neurology referrals, with a sustained $30 \%$ reduction in admissions to the ward team and an increase in the zero length-of-stay rate from $25 \%$ to $40 \%$.

We fully support the conclusions reached by the St George's team and agree that a proactive acute neurology model of care can have a transformative effect. Our experience of running an acute neurology service demonstrates these services remain both efficient and sustainable over a prolonged period of time.

STUART WEATHERBY

Consultant neurologist, University Hospitals Plymouth NHS Trust, Plymouth, UK

MARTIN SADLER

Consultant neurologist, University Hospitals Plymouth NHS Trust, Plymouth, UK

SIMON EDWARDS

Consultant neurologist, University Hospitals Plymouth NHS Trust, Plymouth, UK

AZLISHAM MOHD NOR

Consultant neurologist, University Hospitals Plymouth NHS Trust, Plymouth, UK

LIZ HOUSEHAM

Consultant neurologist, University Hospitals Plymouth NHS Trust, Plymouth, UK

DANIEL LASHLEY

Consultant neurologist, University Hospitals Plymouth NHS Trust, Plymouth, UK

ALEX SHAH

Consultant neurologist, University Hospitals Plymouth NHS Trust, Plymouth, UK MIKE O'GARA

Consultant neurologist, University Hospitals Plymouth NHS Trust, Plymouth, UK

EINMAN ABDELGADIR

Consultant neurologist, University Hospitals Plymouth NHS Trust, Plymouth, UK 